\title{
Fast Convergence and Improved Particle Swarm Hybrid Optimization Algorithm
}

\author{
Li Yi-ran ${ }^{1}$ and Zhang Chun-na ${ }^{2}$ \\ ${ }^{1}$ College of Applied Technology, University of Science and Technology Liaoning, \\ Anshan Liaoning 114011, China \\ ${ }^{2}$ School of Software, University of Science and Technology Liaoning, Anshan \\ Liaoning 114051, China
}

\begin{abstract}
Aiming at the problem that the particle in the traditional particle swarm optimization algorithm is easy to fall into the local optimum and the convergence rate is slow, this paper proposed an improved particle swarm optimization algorithm. In particle swarm optimization algorithm, the advantages and disadvantages of the algorithm is directly decided by the performance of the particle, the paper introduced the chaos mechanism, enhance the ergodicity and particle will be quantized in the solution space, on the premise of ensuring diversity of solution, the particle get better global search ability. Meanwhile, based on the problem of slow convergence speed of the algorithm in the late, on the one hand to dynamically adjust the inertia weight of impact speed, makes the particle movement speed tend to be reasonable, on the other hand, using $k$-means algorithm to optimize progeny particle and get more reasonable clustering center, make the algorithm fast convergence. Experiments show that using improved Particle Swarm Optimization algorithm with high precision, strong stability and fast convergence.
\end{abstract}

Keywords: Particle Swarm Optimization algorithm, chaotic sequence, inertia weight, hybrid algorithm

\section{Introduction}

Particle Swarm Optimization algorithm is an evolutionary algorithm for simulating bird foraging behavior, was proposed in 1995 by Kennedy and Eberhart, particle in the solution space is the core of the algorithm followed the running track of optimal particle, ultimately, to find the optimal solution [1-3]. As algorithm has the advantage of simple structure, less arguments and no gradient information, algorithm received extensive attention of people when it just put forward and has been applied in many fields such as function optimization, pattern recognition, neural network, fuzzy system control and so on [4].

Based on the features of Particle Swarm Optimization algorithm, the iterative process of the algorithm is also the process of particle optimization. There are two problems need to pay attention to: (1) the particle is trapped in a local optimum and unable get the global optimal solution; (2) in the late stage of the algorithm, the particle of the poor performance easily form oscillation which leads to stagnation, easily cause the decline in rate of convergence, affect the overall effect of the algorithm.

In order to improve the accuracy and convergence rate, this paper proposed an improved hybrid optimization algorithm based on quantum particle swarm algorithm, namely fusion K-means algorithm in the Particle Swarm Optimization algorithm, and to adjust the K-means, using the shortest path strategy, so that the particle get better performance [5-7], at the same time, the chaos mechanism and quantum theory are introduced to keep the diversity of the population, the algorithm has less overall parameters, easily control and strong ability of global search. Aiming at the problem of 
particle oscillation caused by late flight, adjust the inertia weight of impact speed, to ensure that the particle to find the optimal solution. The analysis of experimental results show that the improved hybrid algorithm compared with other algorithms, effectively improve the performance, strong stability, fast convergence speed and high precision.

\section{The Problems Associated with Particle Swarm Optimization Algorithm}

\subsection{Basic Particle Swarm Optimization Algorithm}

Particle Swarm Optimization algorithm is the simulated evolution process that the optimal solution is obtained through inter individual communication and collaboration, among them, each particle two properties includes two properties of speed and position, the solution of the problem corresponds to the current position of the particle, whose performance depends on the fitness value of the optimization function [8]. In $Q$ dimension space, the particle is initialized to $\left\{x_{1}, x_{2}, \cdots, x_{n}\right\}$, the velocity of the ${ }^{i}$-th particle in the space is defined as $v_{i}=\left\{v_{i 1}, v_{i 1}, \cdots, v_{i Q}\right\}$, the position is defined as $x_{i}=\left\{x_{i 1}, x_{i 1}, \cdots, x_{i Q}\right\}, i=1,2, \cdots, n$, the direction of travel followed by the trajectory of the best particle, here, a particle to search the current optimal position is $P b$, the optimal position of the whole population is $G b$. With the iteration of the algorithm, the position of the particle is changed repeatedly until the termination condition is satisfied, and the optimal solution is obtained. For $k+1$ iterations, each particle updates its own speed and position according to the following formula:

$$
\begin{gathered}
v_{i q}^{k+1}=\alpha v_{i q}^{k}+\beta^{0}\left(x_{P b}-x_{i q}^{k}\right)+\beta^{1}\left(x_{G b}-x_{i q}^{k}\right) \\
x_{i q}^{k+1}=x_{i q}^{k}+v_{i q}^{k+1}
\end{gathered}
$$

In the formula, $\alpha$ is the coefficient of inertia weight, which is used to balance the relationship of travel speed between the current particle. $\beta^{0}, \beta^{1} \in[0,1]$, random distribution. $q=1,2, \cdots, Q$.

On the clustering problem, the vector in $Q$ dimension space is ultimately comes down to getting the division of a class, the definition of the classification is $C$, it need to meet the following formula:

$$
\left\{\begin{array}{l}
C_{i} \neq \phi \\
C_{i} C_{j}=\phi \\
k \\
U_{i=1}^{k}=X
\end{array}\right.
$$

In the formula, $C_{i}, C_{j} \in C, i, j \in[1, k]$, according to this, the degree of dispersion is obtained.

$$
J=\sum_{j=1}^{k} \sum_{X_{i} \in C} D\left(X_{i}, Z_{i}\right)
$$

In the formula, $k$ is the number of clusters, $Z_{i}$ is the $i$-th cluster center, $D\left(X_{i}, Z_{i}\right)$ represents the distance from the sample to the corresponding cluster center.

The premature problem of algorithm mainly reflected in the convergence of the algorithm and the diversity of particle, the particle trajectory is not randomized, in the iterative process, some of the particle with the poor performance will inevitably appear, its speed dropped significantly, or there is an oscillation in a spatial dimension, it is easy 
to fall into the local optimum, and appeared premature. Based on this, this paper puts forward the following several improvements to the performance of the particle, first of all, the chaos mechanism is introduced into the algorithm to enhance the ergodicity of the particle. Furthermore, using the quantum theory to improve the energy of the particle and enhance the ability to find the best. At the same time, the K-means algorithm is used to update the particle state in order to obtain better particle performance.

\subsection{Chaotic Sequence}

Chaos is widely exists in nature, is a kind of nonlinear phenomenon, due to its sensitivity to initial conditions, and has the characteristics of randomness, ergodicity and regularity, therefore, it can search the internal all state in accordance with the laws of its own in a given area, and do not repeat [9-10]. Based on this, using chaos mechanism to optimize the optimization process of particle, the search procedure is as follows:

(1) Defining the initial region, set $N$ dimensional initial vector $R_{0}=\left(R_{01}, R_{02}, \cdots, R_{0 N}\right)$, each value adjacent in $R_{0}$, and the difference is very small.

(2) using the logistics equation calculate the initial vector $R_{0}$, generate chaotic sequence $c_{1}, c_{2}, \cdots, c_{n}$ 。 here, after several iterations, the system will be completely in a state of chaos. Vector layer can be expressed as:

$$
c_{i+1}=c_{i}\left(1-c_{i-1}\right) \lambda
$$

In the formula, $\lambda$ is an iterative control parameters.

(3) Set space particle ${ }^{X_{i}}$, using the formula (3) get better position of ${ }^{X_{i}}$, recorded as ${ }^{X_{i}}$.

$$
X_{i}^{\prime}=r \cdot r n d \cdot c_{j}+X_{i}
$$

In the formula, $r$ is the active radius of the particle $X_{i}, r n d \in[-1,1], j \in[0, n]$.

Mainly ideas of Particle Swarm Optimization algorithm based on chaotic mechanism are reflected in the following aspects: on the one hand, using chaotic sequence to initialize the position and velocity of the particle, as it has the characteristics of ergodicity, not only maintain the diversity of particle, but also enhance the search ability of the particle. Furthermore, the chaotic state can make the particle motion have a continuous.

Particle chaos initialization: the initial value of $X_{i}$ in the formula (6) is given, and modified the velocity of the particle swarm iteration:

$$
\begin{aligned}
v_{i, j}^{\prime}(t+1)= & a v^{\prime \prime}(t)+b^{0}(t)\left(x_{l b}(t)-x_{i, j}(t)\right) \\
& +b^{1}(t)\left(x_{g b}(t)-x_{i, j}(t)\right)
\end{aligned}
$$

In the formula, $a$ is constant in $(0,1], b$ is the random number of normal distribution $[0,1], i \hat{\mathrm{I}}[1, n], \quad j \hat{\mathrm{I}}[1, m], n$ is the number of particle, $m$ is the dimension of space. For $v^{\prime \prime}(t)$ :

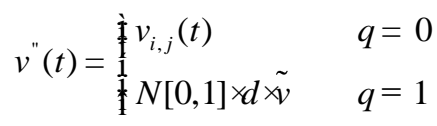

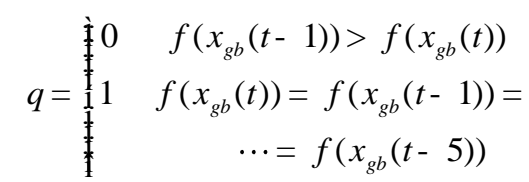

In the formula, $\tilde{v}=v_{\max } x_{i} / 1.1, d=f\left(x_{g b}\right)-f\left(x_{T}\right), c_{i}$ is the new chaotic sequence, $f\left(x_{g b}\right)$ is the satisfactory solution, $f\left(x_{T}\right)$ is the target solution. 
Introduction of chaos mechanism will have a positive impact on the search process, this paper intends to replace the weight coefficient of the random distribution in PSO using the chaotic sequence to update the particle velocity.

\subsection{Inertia Weight}

In this paper introduce the chaos mechanism to improve the accuracy of the algorithm, and for the problem of early easy to early maturity and later evolved slowly suitable improve the inertia weight in the velocity update. The evolution process of particle with the ultimate aim to find the global optimal solution, changes the position and velocity of each iteration, and the position is determined by speed, so the speed is the key of guideline particle flight and optimization algorithm design. Here, the size of the inertia weight directly affects the speed and direction of the particle. Based on the above analysis, this paper intends to adjust the inertia weight of impact speed, to make it do the cosine rule changes according to the evolution algebra. In this way, early the algorithm keep the large inertia weight $\alpha$ in order to improve the local searching ability of the particle, and related expression is as follows:

$$
\alpha=\alpha_{0}+\left(\alpha_{1}-\alpha_{0}\right) \cdot \cos \left(\frac{i_{0} \pi}{2 i_{1}}\right)
$$

In the formula, $\alpha_{0}, \alpha_{1}$ respectively represent the minimum and the maximum inertia weight, $\alpha \in\left[\alpha_{0}, \alpha_{1}\right], \quad i_{0}, i_{1}$ respectively represent the current iteration number and the maximum number of allowable iteration.

\section{The Quantum-Behaved Particle Swarm Hybrid Algorithm}

\subsection{The Quantum-Behaved Particle Swarm}

Quantum-behaved particle swarm embodies the characteristics of superposition state and the expression of probability in quantum theory [11-13]. The combined effect of the two can make the particle appear more states, and then improve the diversity of the population [14]. Among them, the probability expression can express the particle by a certain probability. In this paper, According to the probability of selecting is set two basic state of the particle $\hat{0}$ and $\hat{1}$.

In the quantum-behaved particle swarm algorithm, particle quantization is that every particle is quantized by quantum bit to represent, the representation forms such as mentioned earlier, $\hat{0}$ and $\hat{1}$. At the same time, this kind of state can be expressed as a mixed state, that is a linear combination of the basic state, as follows:

$$
\phi=\lambda_{1} \hat{0}+\lambda_{2} \hat{1}
$$

In the formula, $\lambda_{1} 、 \lambda_{2}$ is a complex number, namely when the quantum state is $\hat{0}$, the probability is $\left.\lambda_{1}\right|^{2}$. When the quantum state is $\hat{1}$, the probability is $\left|\lambda_{2}\right|^{2}$.

The space expression of the quantum state can be evolved into the following formula:

$$
\phi=\cos \hat{\theta 0}+\sin \theta \hat{1}
$$

In the formula, $\theta$ is the phase of quantum state, meet the relationship: $\theta=\arctan \frac{\lambda_{2}}{\lambda_{1}}$.

Thus, after the quantum particle is put into a representation by the wave function quantum space, in which particle can free search the optimal solution, its state without being limited by the vector of speed and position. The corresponding wave function that represent the particle state is as follows: 


$$
\int_{-\infty}^{+\infty} L_{p} d x d y d z=1
$$

The iterative formula of particle is obtained by the Monte Carlo algorithm transforms the quantum state:

$$
\begin{gathered}
P_{m b}=\frac{1}{l} \sum_{i=1}^{l} P_{i} \\
P_{m k}=\theta P_{i k}+(1-\theta) P_{g k} \\
L_{p}(t+1)=P_{i k} \pm a\left|P_{m k}-L_{p}(t)\right| \ln ^{\frac{1}{\lambda}}
\end{gathered}
$$

Above formula, $P_{i}$ is the current position of the particle, ${ }^{P_{b}}$ is the global extremum, $P_{m b}$ is the optimal median, ${ }^{l}$ is the number of particle, ${ }_{m k}$ is a random point between ${ }_{i k}$ and $P_{g k}, a$ is the expansion contraction factor, is a quantitative index, used to control the convergence rate of the algorithm, take the random number between $(0,1)$ in the iteration, if $a>0.5$, then take a positive, or take a negative value.

\subsection{Coding and Fitness Value Calculation}

Particle Swarm Optimization algorithm usually adopts real number coding, and a code corresponding to a feasible solution, this paper uses the way that based on the cluster center, namely a particle corresponding to a set of cluster centers, the position of each particle is composed of $k$ cluster centers, the pretreatment process is that the sample vector of dimension $\mathrm{Q}$ clustering into $k$ classes, the position of particle can be represented as a vector of $k \times Q$, for the same structure, the velocity of particle can also be expressed as the same, the fitness function is defined as $F$, in this way, the corresponding encoding of a particle can be expressed as $X_{i}=\left\{Z_{i 1}, Z_{i 2}, \cdots, Z_{i k}, V_{i 1}, V_{i 2}, \cdots, V_{i k}, F_{i}(X)\right\}$, among them, $Z_{i k}$ is the ${ }^{k}$-th cluster center. The goal of clustering is to obtain the minimal distance between all kinds of samples, using the Euclidean distance to measure, the fitness function is defined as follows:

$$
F(X)=\frac{1}{1+J}
$$

In this paper, the nearest neighbor method is used to calculate the fitness value, the following is a detailed procedure:

Step 1: Particle clustering based on nearest neighbor classification method.

Step 2: By using the clustering results, and combining the formula (4) to calculate the degree of dispersion;

Step 3: The individual fitness and the degree of dispersion are negatively correlated, which is calculated by $f(x)=\frac{t}{J}$, in the formula, $t$ is constant.

\subsection{The Shortest Path $K$ - Means Clustering}

Traditional K-means algorithm uses the Euclidean distance to connect two points, usually for a straight line distance, but in the sample space, there will be a barrier and broken line between two points, so this paper redefines $D^{\prime}(a, b)$ as the shortest path between any two points $a, b$, calculation and analysis steps are as follows:

Step 1: If between two points ${ }^{a, b}$ can be connected by a straight line, then the shortest path is a straight line distance, that is $D^{\prime}(a, b)=D(a, b)$. 
Step 2: Take the ${ }^{a}$ point as the starting point, and all visible blocking points around it are denoted as $B=\left\{p_{1}, p_{2}, \cdots p_{n}\right\}$, calculate the straight line distance from all the blocking points to the ${ }^{a}$ point.

Step 3: Each point as the starting point in the blocking point set $B$ connect the terminal point $b$ get all visible blocking point $B^{\prime}$, and so on, until it is able to connect to the $b$ point in a straight line, thus obtains the shortest distance $D^{\prime}\left(a, p_{i}\right)+D^{\prime}\left(p_{i}+b\right)$ from point $a$ to point $b$.

\subsection{Algorithm Analysis}

The basic idea of the PSO and K-means hybrid algorithm is to use the PSO algorithm to evolve a number of particle and form a particle swarm, by K-means optimize the new generation particle in the evolution of particle to obtain the optimal particle, iteration loop until the termination condition of the algorithm is satisfied. In this way, not only solved the PSO algorithm sensitivity to initial conditions, the particle is not easy to fall into local optimum, at the same time, due to the introduction of $\mathrm{K}$ - means algorithm, making the overall convergence of the hybrid algorithm has been very good improvement, achieve the purpose of fast convergence, the concrete steps of the hybrid algorithm are as follows:

Step 1: Population initialization, randomly selected ${ }^{k}$ particle as cluster center in $n$ particle, $k \leq n$, set the position and velocity.

Step 2: According to the nearest neighbor principle, the population cluster division is implemented by using the current clustering center particle, and the fitness value is calculated by the formula (17).

Step 3: The fitness value of each particle is extracted, and compared with the optimal position $\mathrm{P}$ which is experienced by the particle, the better is updated.

Step 4: According to the formula (1) and (2), and refer to the formula (6) and (7) to update the position and velocity of the particle.

Step 5: The improved K-means algorithm is used to optimize the newly generated particle, intends to recalculate the population cluster centers and carry on the reasonable division, then update the fitness value of the particle to replace the original individual codes.

Step 6: Judge whether the iteration meet the termination conditions, if the termination condition is satisfied, the algorithm will be stopped, otherwise the algorithm will jump to step 2.

\section{Experimental Analysis}

According to the focus of this paper, the experiment is divided into three parts: optimization ability, convergence ability and the analysis of clustering results. In optimization ability, this paper select five benchmark functions to complete the test, respectively is Schwefel , Rosenbrock , Noncontinuous Rastrigin , Griewank , Generalized Penalized. Among them, (1) and (2) is a single mode function, only one extreme point is defined within the interval, which is used to analyze the precision and performance of the algorithm. (3), (4) and (5) is a multi modal function, there are multiple extreme points in the interval, which is mainly used to test the global search capability of the algorithm, the following are five benchmark function definitions and related parameters.

(1) Schwefel function formula is as follows:

$$
\operatorname{Schwefel}(x)=\stackrel{\mathrm{a}}{i=1}_{D}^{D}\left(x_{i} \sin \sqrt{\left|x_{i}\right|}\right)
$$

(2) Rosenbrock function formula is as follows: 


$$
\operatorname{Rosenbrock}(x)=\stackrel{̊}{\mathrm{D}}_{i=1}^{D}\left(100\left(x_{i+1}-x_{i}^{2}\right)^{2}+\left(1-x_{i}\right)^{2}\right)
$$

(3) Noncontinuous Rastrigin function formula is as follows:

$$
\begin{aligned}
& \text { Noncontinuous Rastrigin }=\underset{i=1}{\stackrel{D}{a}}\left[x_{i}^{2}-10 \cos 2 p x_{i}+10\right] \\
& x_{i}=\begin{array}{lll}
k_{i} & \left|k_{i}\right|<0.5 \\
\frac{1}{1} \operatorname{round}\left(2 k_{i}\right) / 2 & \left|k_{i}\right|^{\beta} & 0.5
\end{array}
\end{aligned}
$$

(4) Griewank function formula is as follows:

$$
\text { Griewank }=\frac{1}{4000} \stackrel{̊}{i=1}^{D}\left(x_{i}\right)^{2}-\stackrel{\tilde{O}}{\mathrm{O}}_{i=1} \cos \left(x_{i} / \sqrt{i}\right)+1
$$

(5) Generalized Penalized function formula is as follows:

$$
\begin{aligned}
& \text { Generalized Penalized }=\frac{p}{D}\left\{10 \sin ^{2}\left(p y_{i}\right)\right. \\
& +\stackrel{\mathrm{a}}{i=1}^{D}\left(y_{i}-1\right)^{2}\left[1+10 \sin ^{2}\left(p y_{i+1}\right)\right. \\
& \left.\left.+\left(y_{D}-1\right)^{2}\right]\right\}+\stackrel{\AA}{\mathrm{a}}_{i=1}^{D} u\left(x_{i}\right) \\
& y_{i}=1+\frac{1}{4}\left(x_{i}+1\right)
\end{aligned}
$$

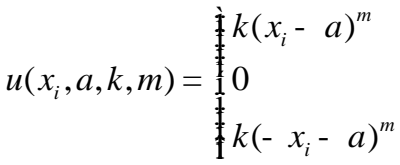

Table 1. Parameter of Benchmark Function

\begin{tabular}{cccc}
\hline Benchmark function name & dimension & $\begin{array}{c}\text { Range of } \\
\text { value }\end{array}$ & optimal value \\
\hline Schwefel & 30 & {$[-10,10]^{D}$} & -12569.5 \\
\hline Rosenbrock & 30 & {$[-10,10]^{D}$} & 0 \\
\hline Noncontinuous Rastrigin & 30 & {$[-5.12,5.12]^{D}$} & 0 \\
\hline Griewank & 30 & {$[-600,600]^{D}$} & 0 \\
\hline Generalized Penalized & 30 & {$[-50,50]^{D}$} & 0 \\
\hline
\end{tabular}

In the experiment, compare and analyze three kinds of algorithms, which are the traditional particle swarm algorithm(PSO), the traditional hybrid particle swarm algorithm(KPSO) and Improved hybrid Particle Swarm Optimization algorithm is proposed in this paper(IK-QPSO). Random test benchmark function, set 100 times, and take the mean, set the population size is 20 , the maximum number of iteration is 1200 . Using two indicators to evaluate the algorithm ability to find optimal solutions: Mean Best Fitness(MBF) and Standard Deviation(SD). Randomly selecting four benchmark functions determine convergence. The clustering effect are analyzed by using the sample data of UCI universal database, data sets are: Vehicle, Wine and Same. Among them, Vehicle and Wine are low dimensional data sets, the former dimension is 18 , the class number is 4 , the number of each sample set is 800 . The latter dimension is 13 , the class number is 3 , the number of each sample set is 150 . Same is a high dimensional data set, the dimension is 16090 , the class number is 3 , the number of each sample set is 300 . The maximum iteration of the algorithm is 200 , using normalized mutual information(NMI) to implement evaluation, which is a kind of clustering effect evaluation method, reactive the similarity between sample clustering results and real category, the value range is set 
to $^{[0,1]}$, the bigger the value, the better the clustering effect is. The following is the corresponding comparison results:

Table 2. Test value of Benchmark Function

\begin{tabular}{ccccc}
\hline Benchmark function & Index type & PSO & SFLA-PSO & ISFLA-QPSO \\
\hline Schwefel & MBF & $1.79 \mathrm{e}-23$ & $1.04 \mathrm{e}-21$ & $1.12 \mathrm{e}-31$ \\
& SD & $2.38 \mathrm{e}-47$ & $4.78 \mathrm{e}-15$ & $1.87 \mathrm{e}-22$ \\
Rosenbrock & MBF & 24.3 & 11.4 & 10.3 \\
Noncontinuous Rastrigin & SD & 26.9 & 14.2 & 10.5 \\
& MBF & 18.4 & 0.41 & 0.44 \\
Griewank & MBF & 7.3 & 0.39 & 0.58 \\
Generalized Penalized & SD & $1.21 \mathrm{e}-17$ & $5.38 \mathrm{e}-32$ & $1.27 \mathrm{e}-44$ \\
& MBF & $6.01 \mathrm{e}-21$ & $1.31 \mathrm{e}-28$ & $6.37 \mathrm{e}-30$ \\
& SD & $1.27 \mathrm{e}-20$ & $1.71 \mathrm{e}-43$ & $1.05 \mathrm{e}-56$ \\
\hline
\end{tabular}

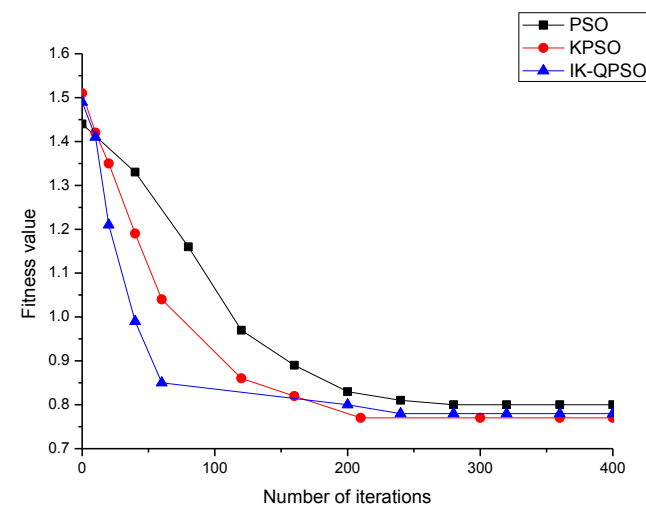

(a) Result of Rosenbrock Function Convergence

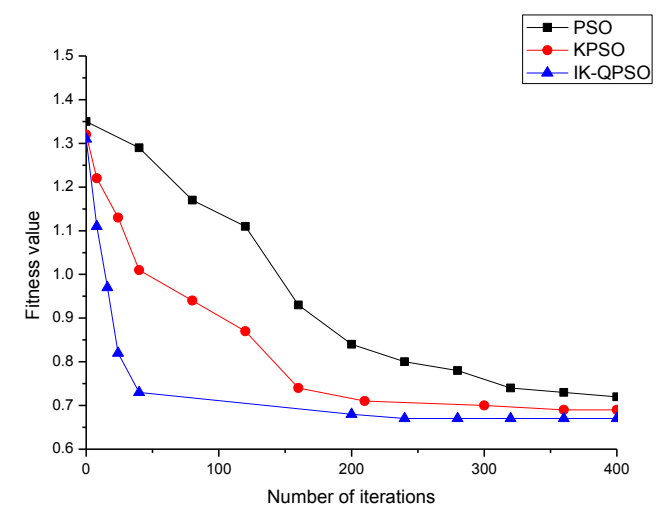

(b) Result of Noncontinuous Rastrigin Function Convergence 


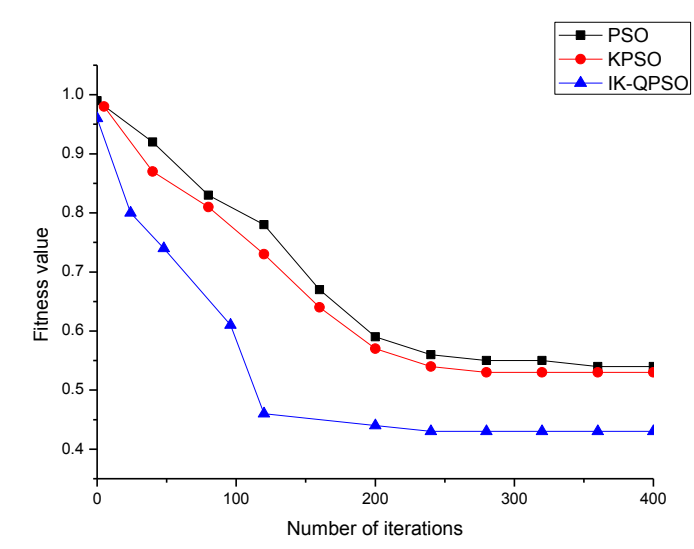

(c) Result of Griewank Function Convergence

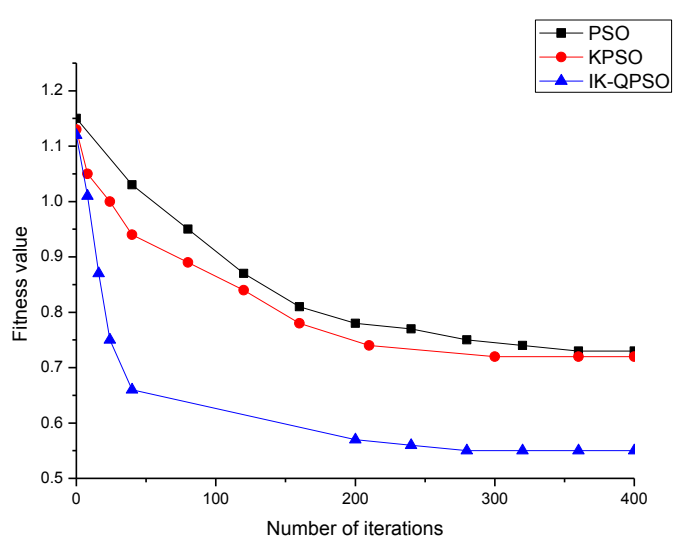

(d) Result of Generalized Penalized Function Convergence

Figure 1. Comparison Result of Benchmark Function Convergence

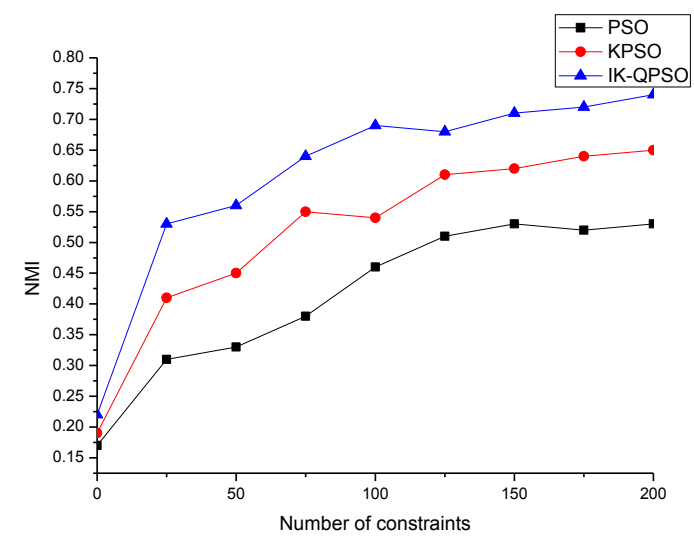

(a) Comparison Results of NMI Value in Vehicle 


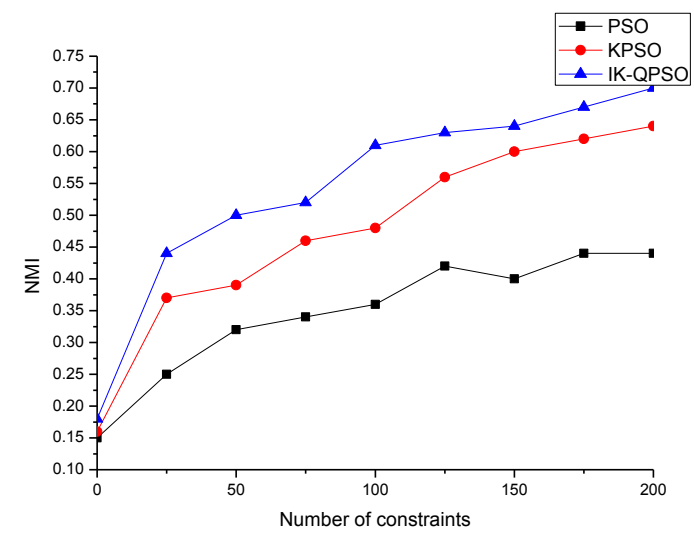

(b) Comparison Results of NMI Value in Wine

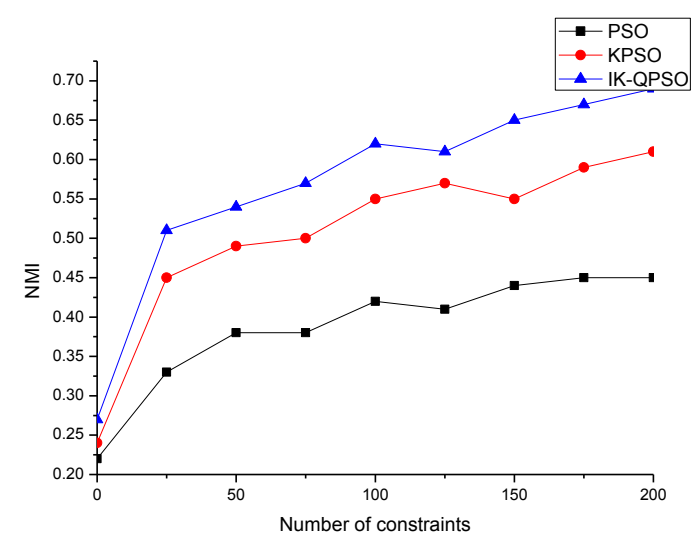

(c) Comparison Results of NMI Value in Same

\section{Figure 2. Comparison Results of NMI Value in Data Set}

Table 1 is the index analysis of the three algorithms, It is not difficult to see from the data, the improved IK-QPSO algorithm both stability and accuracy compared with the other two algorithms have obvious advantages, mostly the chaos mechanism is introduced into the algorithm to enhance the particle activity, considering the problem of poor particle activity in the later period, on the one hand, adjust the speed weight, on the other hand, the combined with the K-means algorithm to optimize particle, not only improve the algorithm global search ability, but also enhance its convergence.

Figure 1 is the index analysis of the three algorithm convergence, in which the improved algorithm affect the convergence curve of (a), (b), (d), the initial decline speed is better, (c) is slightly inferior, but the convergence speed at the late period of three can reflect the algorithm advantage at the late period. Here, the fast convergence of the algorithm is mainly the fusion of chaos and quantum theory, which enhance the performance of the particle, especially in the latter stage of the algorithm is particularly evident, with the gradual operation of the algorithm, the performance of particle decreased in the traditional particle swarm algorithm, it is easy to fall into local optimal bound, the improved algorithm uses the cosine to optimize the inertia weight of the impact speed, and the improved K-means algorithm is used to recalculate the cluster center, to improve the performance of poor particle activity, therefore, it can be seen from Figure 1, in addition to (a) the curve is slightly insufficient, the convergence of the improved algorithm of the other three benchmark functions is improved effectively.

Figure 2 is the index analysis of three kinds of data sources in UCI data set, it can be seen that the improved algorithm in the three algorithms is obviously better than the other 
two algorithms from the experimental data. In the initial stage of the algorithm, when the iteration algebra is 0, the NMI values of the three algorithms are basically the same. With the algorithm running, the number of iterations is increase, PSO algorithm in three figure because there is no improvement will fall behind the other improved algorithm. By comparison, the performance index of IK-QPSO is the best. The improvement effect can be reflected when the iteration number reach 125, the NMI value of other two algorithm will have a certain oscillation, but IK-QPSO continued to rise, especially at the end of the algorithm is still maintained the ascent, it shown that the improvement objectives.

\section{Conclusions}

This paper proposed a hybrid algorithm that Particle Swarm Optimization algorithm combined with the $\mathrm{K}$ - means algorithm, in dealing with Particle Swarm Optimization algorithm is easy to fall into local optimum and slow convergence speed of the late effect is better, and the accuracy of the algorithm also has certain range to improve. Based on particle swarm algorithm is easy to fall into local optimal problems, to adjust the inertia weight. At the same time introducing chaos mechanism to improve the global search ability of particle, and quantum particle, effectively regulate the particle movement speed, to improve the diversity of solution. In terms of convergence improvement, using particle of k-means algorithm in the evolution is proposed in this paper, to get the new clustering center, which combined with the Particle Swarm Optimization algorithm, can make the algorithm to get fast convergence. Experimental results show that the improved algorithm in precision, stability and convergence has been improved.

\section{References}

[1] A. Khare and S. Rangnekar, "A review of particle swarm optimization and its applications in Solar Photovoltaic system", Applied Soft Computing, vol. 13, no. 5, (2013), pp. 2997-3006.

[2] H. Garg, "A hybrid PSO-GA algorithm for constrained optimization problems", Applied Mathematics and Computation, vol. 274, (2016), pp. 292-305.

[3] E. V. Kumara, G. S. Raajab and J. Jerome, "Adaptive PSO for optimal LQR tracking control of 2 DoF laboratory helicopter", Applied Soft Computing, vol. 41, (2016), pp. 77-90.

[4] R. Ugolotti and S. Cagnoni, "Automatic Tuning of Standard PSO Versions", Proceedings of the Companion Publication of the 2015 Annual Conference on Genetic and Evolutionary Computation, ACM, (2015), pp. 1501-1502.

[5] A. M. Lal and S. M. Anouncia, "Semi-supervised change detection approach combining sparse fusion and constrained k means for multi-temporal remote sensing images", The Egyptian Journal of Remote Sensing and Space Science, vol. 18, no. 2, (2015), pp. 279-288.

[6] N. Tsapanos, A. Tefas, N. Nikolaidis and I. Pitas, "A novel defect prediction method for web pages using k-means++", Expert Systems with Applications, vol. 42, no. 19, pp. 6496-6506.

[7] A. Broder, L. G. Pueyo, V. Josifovski, S. Vassilvitskii and S. Venkatesan, "Scalable K-Means by ranked retrieval", Proceedings of the 7th ACM international conference on Web search and data mining, ACM, (2014), pp. 233-242.

[8] A. Mekhmoukh and K. Mokrani, "Improved Fuzzy C-Means based Particle Swarm Optimization (PSO) initialization and outlier rejection with level set methods for MR brain image segmentation", Computer Methods and Programs in Biomedicine, vol. 122, no. 2, (2015), pp. 266-281.

[9] L. D. S. Coelho and V. C. Mariani, "Use of chaotic sequences in a biologically inspired algorithm for engineering design optimization”, Expert Systems with Applications, vol. 34, no. 3, (2008), pp. 19051913.

[10] E. Araujo and L. D. S. Coelho, "Particle swarm approaches using Lozi map chaotic sequences to fuzzy modelling of an experimental thermal-vacuum system", Journal Applied Soft Computing, vol. 8, no. 4, (2008), pp. 1354-1364.

[11] A. Bagheria, H. M. Peyhanib and M. Akbaric, "Financial forecasting using ANFIS networks with Quantum-behaved Particle Swarm Optimization”, Expert Systems with Applications, vol. 41, no. 14, (2014), pp. 6235-6250.

[12] M. Helbig and A. P. Engelbrecht, "The Effect of Quantum and Charged Particles on the Performance of the Dynamic Vector-evaluated Particle Swarm Optimization Algorithm", Proceedings of the 2015 Annual Conference on Genetic and Evolutionary Computation, ACM, (2015), pp. 25-32.

[13] B. Haddara, M. Khemakhema, S. Hanafib and C. Wilbaut, "A hybrid heuristic for the 0-1 Knapsack Sharing Problem”, Expert Systems with Applications, vol. 42, no. 10, (2015), pp. 4653-4666. 
[14] N. H. Sweilama, A. A. Tharwatb and N. K. A. Moniem, "Support vector machine for diagnosis cancer disease: A comparative study”, Egyptian Informatics Journal, vol. 11, no. 2, (2010), pp. 81-92.

\section{Authors}

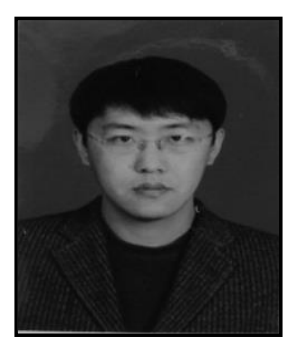

Y. R. Li, received the Master's degree in computer application technology from University of Science and Technology Liaoning, in 2008. Currently, he is a lecturer at School of applied technology college at University of Science and Technology Liaoning. His research interests include Distributed computing and data mining.

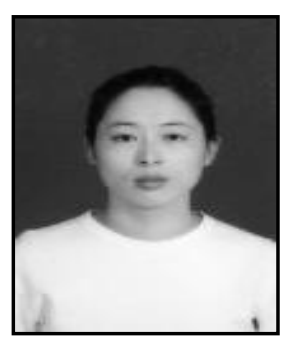

C. N. Zhang, received the Master's degree in computer application technology from University of Science and Technology Liaoning, in 2007. Currently, she is a lecturer at School of Software Engineering at University of Science and Technology Liaoning. Her research interests include Distributed computing and data mining. 